\title{
К ВОПРОСУ ЭПИДЕМИОЛОГИИ И ДИАГНОСТИКИ ОСТРОГО АППЕНДИЦИТА
}

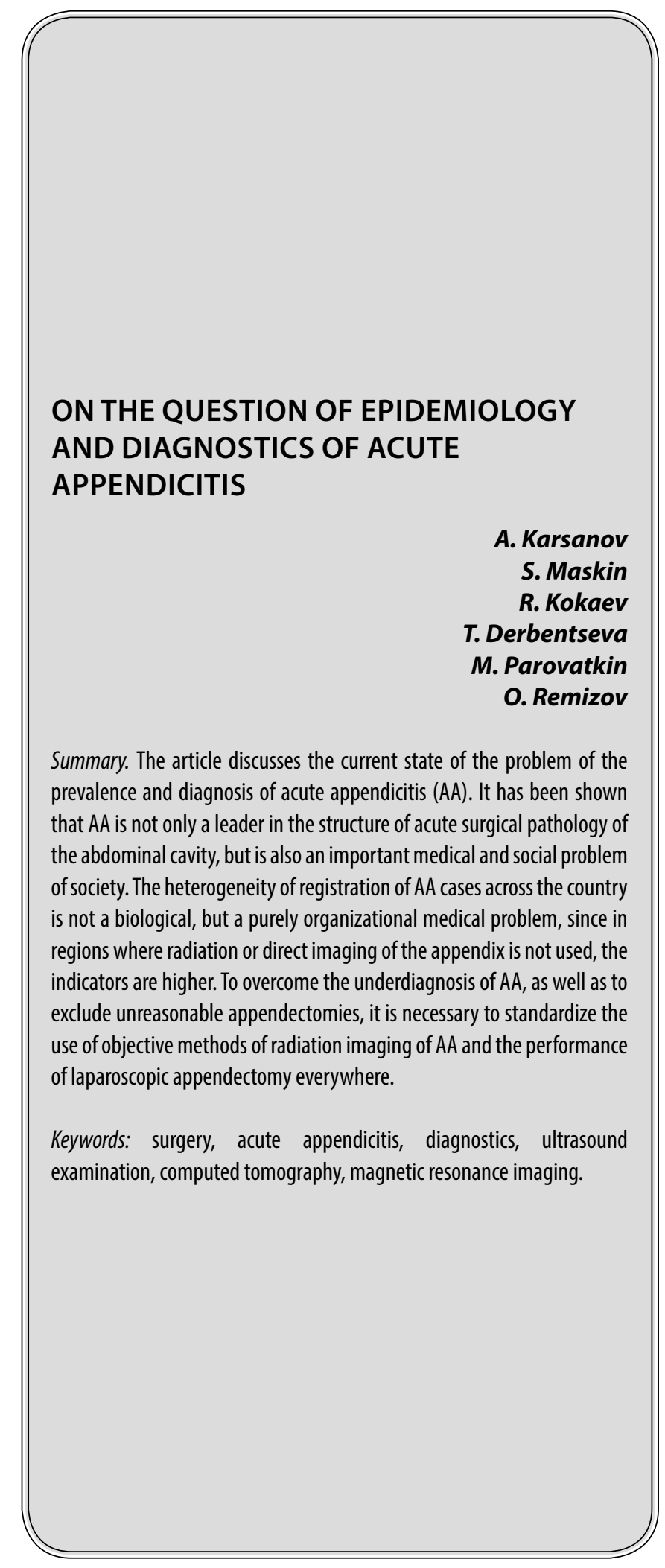

Карсанов Алан Мухарбекович К.м.н., дочент, ФГБОУ ВО «Северо-Осетинская государственная медицинская академия»

(2. Владикавказ)

karsan@inbox.ru

Маскин Сергей Сергеевич

Д.м.н., профессор, ФГБОУ ВО «Волгоградский государственный медицинский университет»

(г. Волгоград)

maskins@bk.ru

Кокаев Роман Игоревич

ФГБОУ ВО «Северо-Осетинская государственная медицинская академия» (2. Владикавказ)

sogma.rso@gmail.com

Дербенцева Татьяна Викторовна

К.м.н., дочент, ФГБОУ ВО «Волгоградский государственный медицинский университет»

(2. Волгоград)

TVDerbentseva@volgmed.ru

Пароваткин Михаил Иванович

К.м.н., дочент, ФГБОУ ВО «Волгоградский государственный

Медицинский университет» (г. Волгоград)

Ремизов Олег Валерьевич

Д.м.н., ректор, ФГБОУ ВО «Северо-Осетинская государственная медицинская академия»

(2. Владикавказ)

oleg_remizov@mail.ru

Аннотация. В статье рассмотрено современное состояние проблемы распространенности и диагностики острого аппендицита (ОА). Показано, что ОА не просто лидирует в структуре острой хирургической патологии брюшной полости, но и является важной медико-социальной проблемой общества. Неоднородность регистрации случаев 0 п по стране является не биологической, а сугубо организационной медицинской проблемой, поскольку в регионах, где не применяется лучевая или прямая визуализация червеобразного отростка показатели выше. Для преодоления гиподиагностики ОА, так же как и для исключения необоснованных аппендэктомий, необходимо повсеместно стандартизировать применение объективных методов лучевой визуализации ОА и выполнение лапароскопической аппендэктомии.

Ключевые слова: хирургия, острый аппендицит, диагностика, ультразвуковое исследование, компьютерная томография, магнитно-резонансная томография. 


\section{Эпихемиология острого аппенАицита}

В структуре контингента больных с острыми абдоминальными заболеваниями в Российской Федерации острый аппендицит (ОА) лидирует [1-3]. Так интенсивный показатель заболеваемости ОА в нашей стране составил в 2018 г. 142,3 на 100 тыс. населения. По этому параметру ОА опережает показатель, рассчитанный для острого холецистита $(139,0)$ и для острого панкреатита $(131,2)[1,2,4]$. Ежегодно в Российской Федерации оперируют 160-170 тысяч пациентов с ОА [3].

Примечательно, что констатированная для ОА заболеваемость в нашей стране отличается существенной неоднородностью. Так в Центральном $(114,7)$ и Северо-Западном $(117,5)$ федеральных округах заболеваемость самая низкая. В то же время в Приволжском $(178,6)$, Дальневосточном $(165,0)$ и Уральском $(163,2)$ федеральных округах выявлены наибольшие показатели заболеваемости ОА [2].

Не вдаваясь в тонкий анализ ситуации, скажем, что в регионах, где наименьшая заболеваемость ОА, наиболее высока доля применения лапароскопического метода лечения ОА $[1,5]$. Так, если в целом по Российской Федерации частота выполнения лапароскопической аппендэктомии по поводу ОА в 2018 г. составила $25,1 \%$, то в Северо-Западном округе она достигает 53,1\%, а в Центральном - 46,8\% из общего числа всех аппендэктомий. На этом фоне контрастно выглядят показатели использования современной технологии хирургического лечения ОА в Приволжском (9,8\%), Дальневосточном (9,9\%) и Северо-Кавказском (15,2\%) федеральных округах [2]. Госпитальная летальность при ОА в 2018 г. в РФ составила 0,14\% [3].

\section{Острый аппенАицит как кАиническая проблема}

С учетом значительного опыта лечения ОА в мире и широкого распространения технологий диагностики и современного лапароскопического лечения нельзя признать, что хирургическое сообщество единодушно в оценке всех аспектов данной клинической проблемы $[1,3-7]$. Это видно хотя бы по тому, что с определенной периодичностью выходят дополненные новыми доказательными данными глобальные и национальные согласительные рекомендации, из которых можно извлечь, помимо важных стратегических для клинической хирургии сведений, акцентированные указания на проблемные аспекты диагностической тактики технологии лечения различных категорий пациентов с ОА $[6,8,9]$.

К таким аспектам можно без сомнения отнести разработку надежных стандартов диагностики и алгоритма ле- чения беременных женщин, детей, пациентов пожилого и старческого возраста, пациентов с декомпенсированными интеркуррентными заболеваниями [5, 8, 10, 11]. Не менее актуален выбор эффективного лечебного подхода при гнойно-септических осложнениях ОА [1, 6, 11, 12].

Речь в данном случае традиционно идет о локализованных и распространенных гнойных перитонитах и абдоминальном сепсисе аппендикулярного генеза, в практических аспектах контроля над источником осложненной внутрибрюшной инфекции, согласно результатам публикаций представителей различных хирургических школ, по-прежнему нет единодушия $[2,8,10,12]$.

Повсеместная широкая распространенность ОА как нозологии и максимальная неоднородность подверженных этому заболеванию пациентов лежат в основе организационных сложностей стандартизации диагностических подходов. Клинические факторы, такие как: плюривисцеральный характер основных симптомом ОА, прежде всего болей в правой половине брюшной полости, и их гетерогенность у разных категорий пациентов, приводят на практике к значительному числу диагностических ошибок $[1,4,6,10,11]$.

Ложноположительные исходы диагностики ОА довольно стойко превышают 13\%, что в абсолютных цифрах составляет значительное число населения. Необоснованные аппендэктомии лежат в основе высокого материально-технического бремени общества на лечение и реабилитацию этих пациентов. С другой стороны, недооценка локальных деструктивных изменений в червеобразном отростке может приводить к фатальным последствиям для конкретного пациента $[2,4,8,10]$.

По-прежнему является предметом дискуссии такой вопрос, как роль и место консервативного лечения ОА у отдельных категорий пациентов, которым рискованно предлагать традиционное либо лапароскопическое оперативное лечение $[5,6,8,12]$. Хотя справедливости ради следует сказать, что на общую стратегию лечения пациентов с ОА, ввиду малого клинического значения такой практики в мире, в том числе и с учетом отдаленных перспектив необходимости планового удаления червеобразного отростка, а это существенно повышает стоимость подобного тактического подхода, концепция консервативного лечения ОА существенно не влияет [6, $8,13]$. Большинство хирургов в мире вполне обоснованно являются сторонниками хирургического лечения ОА.

Компоненты и заАачи неинвазивной АИагностикИ острого аппенАицита

Несмотря на всеобщее понимание профессиональным сообществом не только медицинской, но и важной 
социально-экономической значимости проблемы ОА, для клиницистов всех поколений диагностика ОА всегда была и продолжает оставаться не простой задачей [1, $5,6,8,10]$. Это в первую очередь обусловлено широкой вариабельностью и разнообразными "масками" клинических проявлений, быстрым и неуклонным развитием грозных осложнений, необходимостью практически в каждом случае подозрения на ОА проводить дифференциальную диагностику с широким набором альтернативных диагнозов $[4,6,8,11]$.

В рамках данной статьи попробуем осветить такой, не всеми однозначно трактуемый аспект диагностики ОА, как роль и место использования современных методов неинвазивной лучевой визуализации (ЛВ). За последние 30 лет ЛВ стала центральным элементом диагностики ОА, снизив как количество необоснованных аппендэктомий, так и расходы на систему здравоохранение всех стран $[6,10,11]$.

Многие современные исследователи связывают повышение точности диагностики у пациентов с болевым синдромом в правой подвздошной области именно с неинвазивными методами ЛВ $[8,10,11,13,14]$. лв:

Для диагностики ОА на сегодня доступны три метода

1. Ультразвуковое исследование (УЗИ);

2. Компьютерная томография (КТ);

3. Магнитно-резонансная томография (МРТ).

Для начала рассмотрим общую последовательность решения стоящих перед любым из методов ЛВ задач, позволяющую надеяться на эффективную верификацию патологических изменений в червеобразном отростке и любых других органах брюшной полости.

Первостепенной задачей является точная визуализация и регистрация местоположения червеобразного отростка и подтверждение воспалительных изменений в нем. Это особенно актуально в третьем триместре беременности, а также при ретроцекальном или ином варианте атипичного расположения аппендикса $[2,10,14]$.

Второй задачей, при первоначальной констатации воспалительной альтерации червеобразного отростка, является выявление потенциальных осложнений ОА в виде аппендикулярного инфильтрата, периаппендикулярного абсцесса, распространенного гнойного перитонита, пилефлебита, ретроперитонеальной флегмоны [2, $7,12,14]$.

Третьей, не всегда акцентированно декларируемой задачей (хотя она должна составлять обязательный элемент диагностического протокола) является исклю- чение альтернативных причин имеющейся у пациента клинической симптоматики. Иными словами - должна быть выполнена инструментальная дифференциальная диагностика синдрома острых абдоминальных болей [1, $6,8,10]$.

\section{Технологические особенности Аиагностики острого аппенАицита}

После клинико-лабораторного обследования трансабдоминальное УЗИ является основным методом диагностики ОА. Однако в сложных для визуализации червеобразного отростка случаях трансвагинальное УзИ значительно повышает ценность метода для верификации заболеваний женской половой сферы, таких как аднексит, симптоматические кисты яичников или внематочная беременность. Оно также должно быть облигатной опцией для диагностики аппендицита, если предыдущее трансабдоминальное УЗИ не позволило убедительно поставить диагноз [6, 14].

Трудности трансабдоминальной ультразвуковой визуализации червеобразного отростка возникают у беременных в третьем триместре. В этом случае оправдано выполнять УзИ комбинированным (трансабдоминальным и трансвагинальным) доступом, что даже значимо снижает необходимость использования других методов ЛВ [14].

В силу технологических особенностей метода диагностическая ценность УЗИ снижается в условиях гиперпневматоза, ожирения, аномальной анатомии и целого рядя других частных особенностей, которые не известны исследователю заранее и трудно преодолимы в ходе самого исследования [15].

По данным L.A. Binkovitz и соавт., суммарная частота установленных по данным УЗИ диагнозов ОА составила 72\% от всех обследованных с подозрением на это заболевание пациентов. Общая точность, чувствительность и специфичность УЗИ в диагностике ОА на детской популяции составил 96,0\%, 94,8\% и 96,3\% соответственно [16].

Последние публикации, посвященные значимости УЗИ для дооперационной верификации ОА достаточно показательны. Согласно метаанализу, опубликованному J. Matthew Fields и соавт., чувствительность и специфичность УЗИ при диагностике ОА составили 91\% и 97\% соответственно [17].

При отсутствии убедительной картины ОА, но сохраняющейся средней вероятности этого диагноза, после УЗИ большинство национальных сообществ рекомендуют применения КТ [6, 8]. Ранее было доказано, что часто- 
та неоправданных аппендэктомий после КТ исследований со сниженной дозой была не чаще $(3,5 \%)$, чем после стандартной методики обследования (3,2\%) [18]. Тем самым, постулат, что принцип безопасности должен быть столь же доминантен, как и стратегическая целесообразность своевременной постановки диагноза ОА, получил свое научное обоснование [19].

На сегодня признано, что МРТ имеет по крайней мере такую же чувствительность и специфичность, что и КТ. Несмотря на более высокую стоимость и проблемы с доступностью во многих центрах, МРТ следует предпочесть рентгеновскому компьютерному исследованию в качестве метода ЛВ у беременных женщин в случаях безуспешных попыток визуализировать червеобразный отросток при УЗИ [20].

Обсуждая целесообразность, последовательность применения, диагностическую ценность и потенциальный вред от того или иного метода ЛВ следует учитывать их диагностическую точность при осуществлении дифференциальной диагностики [21]. Так УЗИ высоко значимо при острых гинекологических заболеваниях, при почечной колике, при остром мезадените. КТ незаменимо при остром панкреатите, почечной колике справа, перфоративном дивертикулите и иных, менее частых острых абдоминальных заболеваниях $[1,6,8]$. В странах, где КТ имеет историю рутинного использования, более высокая частота раннего выявления опухолевой патологии брюшной полости и иных малосимптомных заболеваний [6] и, применительно к обсуждаемому вопросу, закономерно реже выполняются необоснованные аппендэктомий [22].

\section{Зак^ючение}

Неотложная абдоминальная хирургия на современном этапе своего развития по-прежнему объединяет широкий спектр заболеваний с высоким риском осложнений, нежелательных исходов и даже летальности. ОА является самой распространенной нозологией в общей структуре острых хирургических заболеваний органов брюшной полости. Частота осложненного течения ОА у различных категорий пациентов достигает 6,5-20\%. Основным методом лечения ОА является хирургический. В нашей стране ежегодно выполняется до 170 тысяч аппендэктомийю

Постоянный научный поиск наиболее современных и результативных подходов к диагностике и лечению ОА отражается в регулярно пополняемых новыми доказательными данными глобальных и национальных согласительных рекомендациях.

Различные методы неинвазивной диагностики ОА получили развитие именно в результате того, что для многих поколений хирургов своевременная диагностика ОА всегда была и продолжает оставаться далеко не простой задачей, а диагностические ошибки несут угрозу здоровью конкретного пациента и усугубляют материальное ремы общества в целом. Этому есть и вполне физиологическое объяснение, основанное на широкой вариабельности клинических "масок" ОА и на необходимости в половине клинических случаев проводить дифференциальную диагностику с широким набором альтернативных диагнозов.

Таким образом, перед визуализационными методами стоят диагностические задачи по топической идентификации червеобразного отростка и подтверждению воспалительных изменений в нем.

Помимо этого, при использовании методов ЛВ должны быть выявлены локальные и дистантные осложнения ОА, исключены альтернативные причины имеющейся у пациента клинической симптоматики.

Современные требования к методам диагностике ОА отражают суть использования этих технологий в рамках общей стратегии недопущения гиподиагностики и следующих за этим рисков тяжелых гнойно-септических осложнений для пациента, а с другой стороны, важно исключить выполнение необоснованных оперативных вмешательств.

\section{ЛИТЕРАТУРА}

1. Острый аппендицит / А.В. Сажин, Т.В. Нечай, А.И. Кириенко. — Москва: 000 «Медицинское информационное агентство», 2019. - 208 с.

2. Ревишвили А.Ш., Сажин В.П., Оловянный В.Е., Захарова М.А. Современные тенденции в неотложной абдоминальной хирургии в Российской Федерации // Хирургия. Журнал им. Н.И. Пирогова. — 2020.— № 7. - С. 6-11.

3. Хирургическая помощь в Российской Федерации. Информационно-аналитический сборник / Под ред. А.Ш. Ревишвили.- М.: ФГБу “НМИЦ хирургии им. А.В. Вишневского", 2019.- 136 с.

4. Карсанов А.М., Ремизов 0.В., Маскин С.С., Кульчиев А.А., Карсанова 3.0. Диагностика сепсиса // Вестник хирургии.— 2016.— № 6. — С. 98-103.

5. Сажин А.В., Курцер М.А., Коноплянников А.Г., Ивахов Г.Б., Панин А.В., Сон Д.А., Шуляк Г.Д., Серебренникова Ю.А. Осложненные формы острого аппендицита у беременных // Хирургия. Журнал им. Н.И. Пирогова. — 2019. — № 4. - С. 15-23.

6. Di Saverio S., Podda M., De Simone B. et. al. Diagnosis and treatment of acute appendicitis: 2020 update of the WSES Jerusalem guidelines // World J. Emerg Surg. 2020.—№ 1.-P. 27. 
7. Маскин С.С., Карсанов А.М., Дербенцева Т.В., Матюхин В.В., Карсанова 3.0. Дифференцированный выбор тактических решений при генерализованной внутрибрюшной инфекции // Московский хирургический журнал.— 2015.— № 1. - C. 36-40.

8. Fugazzola P., Ceresoli M., Agnoletti V. at al. The SIFIPAC/WSES/SICG/SIMEU guidelines for diagnosis and treatment of acute appendicitis in the elderly (2019 edition) // World J. Emerg Surg. — 2020.— № 1.—P. 19.

9. Карсанов А.М., Маскин С.С., Слепушкин В.Д., Карсанова 3.0., Дербенцева Т.В., Саламова Ф.Т., Караев Т.Р. Клинико-эпидемиологическое значение системного воспаления и сепсиса // Вестник хирургии. — 2015.— № 4.— С. 99-103.

10. Monsonis B., Mandoul C., Millet I., Taourel P. Imaging of appendicitis: Tips and tricks // Eur. J. Radiol. — 2020 — Vol. 130:109165. doi: 10.1016/j.ejrad.2020.109165.

11. Mostbeck G., Adam E.J., Nielsen M.B. et al. How to diagnose acute appendicitis: ultrasound first // Insights Imaging. — 2016. — № 2.— P. $255-263$.

12. Карсанов А.М., Кульчиев А.А., Караев Т.Р., Кокаев И.П., Вахоцкий В.В. Роль современных методов лучевой визуализации при внутрибрюшных гнойно-воспалительных осложнениях толстокишечного генеза // Хирургия. Журнал им. Н.И. Пирогова.— 2015.— № 5.— С. 75-79.

13. Salminen P., Paajanen H., Rautio T. et al. Antibiotic therapy vs appendectomy for treatment of uncomplicated acute appendicitis: the APPAC randomized clinical trial // JAMA. - 2015. — № 23.- P. 2340-2348.

14. Karul M., Berliner C., Keller S., Tsui T.Y., Yamamura J. Imaging of appendicitis in adults // Rofo.— 2014.— № 6. — P. 551-558.

15. Rosenkrantz A.B., Labib A., Ginocchio L.A., Babb J.S. Evaluation for suspected acute appendicitis in the emergency department setting: a comparison of outcomes among three imaging pathways // Clin. Imaging.—2016.— № 4.—P. 788-792.

16. Binkovitz L.A., Unsdorfer K.M., Thapa P. et al. Pediatric appendiceal ultrasound: accuracy, determinacy and clinical outcomes // Pediatr. Radiol.— 2015.— № 13.— P. 1934-1944.

17. Matthew Fields J., Davis J., Alsup C. et al. Accuracy of point-of-care ultrasonography for diagnosing acute appendicitis: a systematic review and and meta-analysis // Acad. Emerg. Med.— 2017.— № 9.—P. 1124-1136.

18. Kim K., Kim Y.H., Kim S.Y. et al. Low-dose abdominal CT for evaluating suspected appendicitis // N. Engl. J. Med.— 2012.— № 366.— P. $1596-1605$.

19. Amitai M.M., Katorza E., Guranda L. et al. Role of emergency magnetic resonance imaging for the workup of suspected appendicitis in pregnant women // Isr. Med. Assoc. J. - 2016. — № 10.-P. 600-604.

20. Sammalkorpi H.E., Mentula P., Savolainen H. et al. The introduction of Adult Appendicitis Score reduced negative appendectomy rate // Scand J. Surg. — 2017. № 3. - P. 196-201.

21. Reddy S.B., Kelleher M., Bokhari S.A.J. et al. A highly sensitive and specific combined clinical and sonographic score to diagnose appendicitis // J. Traum. Acute Care Surg. - 2017.— № 4.- P. 643-649.

22. Andersson M., Kolodziej B., Andersson R.E. et al. Randomized clinical trial of appendicitis inflammatory response score-based management of patients with suspected appendicitis // Br.J. Surg.— 2017.— № 11.—P. 1451-1461.

( К Карсанов Алан Мухарбекович ( karsan@inbox.ru ), Маскин Сергей Сергеевич ( maskins@bk.ru ), Кокаев Роман Игоревич ( sogma.rso@gmail.com ), Дербенцева Татьяна Викторовна (TVDerbentseva@volgmed.ru ),

Пароваткин Михаил Иванович, Ремизов Олег Валерьевич ( oleg_remizov@mail.ru ).

Журнал «Современная наука: актуальные проблемы теории и практики» 